\title{
CENTRALIZING MAPPINGS ON VON NEUMANN ALGEBRAS
}

\author{
MATEJ BREŚAR
}

(Communicated by Palle E. T. Jorgensen)

\begin{abstract}
Let $R$ be a ring with center $Z(R)$. A mapping $F$ of $R$ into itself is called centralizing if $F(x) x-x F(x) \in Z(R)$ for all $x \in R$. The main result of this paper states that every additive centralizing mapping $F$ on a von Neumann algebra $R$ is of the form $F(x)=c x+\zeta(x), x \in R$, where $c \in Z(R)$ and $\zeta$ is an additive mapping from $R$ into $Z(R)$. We also consider $\alpha$-derivations and some related mappings, which are centralizing on rings and Banach algebras
\end{abstract}

\section{INTRODUCTION}

Let $R$ be a ring. Recall that $R$ is called prime if $a R b=0$ implies $a=0$ or $b=0 . R$ is called semiprime if $a R a=0$ implies $a=0$. If $2 x \neq 0$ for every nonzero $x \in R$, then $R$ is said to be 2-torsionfree. The center of a ring $R$ will be denoted by $Z(R)$. We write $[u, v]$ for the commutator $u v-v u$.

A mapping $F$ of a ring $R$ into itself is called centralizing if $[F(x), x] \in$ $Z(R)$ for all $x \in R$. In the special case where $[F(x), x]=0$ for all $x \in R$, the mapping $F$ is said to be commuting. The classical result of E. Posner [19] states that zero is the only centralizing derivation on a noncommutative prime ring. J. Mayne [15] proved the analogous result for centralizing automorphisms on prime rings. A number of algebraists have extended these theorems of Posner and Mayne in several ways (see, e.g., $[1,2,3,6,7,9,12,16,17])$. There has also been some interest in centralizing mappings defined on Banach algebras. In [18] C. R. Miers studied centralizing automorphisms of von Neumann algebras and derivations $d$ of $C^{*}$-algebras with the property that $p(d)$ is centralizing for some complex polynomial $p$. In [5] it was shown that a continuous commuting derivation on a Banach algebra $A$ maps $A$ into its radical.

The authors of the aformentioned references have studied centralizing derivations, endomorphisms, and some related additive mappings. In [4] we described all additive mappings which are centralizing on prime rings $R$ of characteristic

Received by the editors December 10, 1989.

1980 Mathematics Subject Classification (1985 Revision). Primary 46L40, 16A72; Secondary 16A12, 46H05, 46H10, 46L10.

Key words and phrases. Centralizing mapping, commuting mapping, derivation, automorphism, $\alpha$-derivation, von Neumann algebra, prime ring, semiprime ring, Banach algebra. 
not 2; we showed, that every such mapping $F$ is of the form $F(x)=\lambda x+\zeta(x)$, where $\lambda$ is an element from the extended centroid of $R$ and $\zeta$ is an additive mapping from $R$ into the extended centroid of $R$ (see [8] or [13] for the notion of the extended centroid of a prime ring). Combining this with the fact that the extended centroid of a prime $C^{*}$-algebra is isomorphic to the complex numbers [14, Proposition 2.5], we see that if $F$ is an additive centralizing mapping on a prime von Neumann algebra $R$, then there exist $c \in Z(R)$ and an additive mapping $\zeta: R \rightarrow Z(R)$ such that $F(x)=c x+\zeta(x)$ for all $x \in R$. The main result of this paper (Theorem 2.1) states that this conclusion holds in arbitrary (not necessarily prime) von Neumann algebra.

It turns out that under rather mild assumptions every additive centralizing mapping on a ring $R$ is, in fact, commuting [4]. In particular this is true if $R$ is a 2-torsionfree semiprime ring. We will use this result several times (in fact we will work only with commuting mappings).

Let $\alpha$ be an automorphism of a ring $R$. An additive mapping $d$ of $R$ into itself is called an $\alpha$-derivation if it satisfies $d(x y)=\alpha(x) d(y)+x d(y)$ for all $x, y \in R$. Note that the mapping $1-\alpha$, where 1 is the identity on $R$, is an $\alpha$-derivation. 1-derivations are, of course, derivations. In $\S 3$ we consider commuting $\alpha$-derivations of semiprime rings (Theorem 3.4). Two applications of this purely algebraic result are presented. The first one is a characterization of centralizing automorphisms on von Neumann algebras (Corollary 3.5)-this result generalizes a theorem of Miers [18, Theorem 5]. As another consequence of Theorem 3.4 we obtain that every (not necessarily continuous) centralizing derivation of a semiprime Banach algebra $A$ maps $A$ into its radical (Corollary 3.7). In the proof of this corollary we will also use a recent, remarkable result of M. Thomas [21], which states that any derivation of a commutative Banach algebra $A$ maps $A$ into its radical.

In $\S 4$ we prove a result concerning centralizing antihomomorphisms.

We use [10] as a general reference for the theory of von Neumann algebras. If $a$ is a nonzero element in a von Neumann algebra $A$, then $0 \neq a a^{*} a \in a R a-$ thus every von Neumann algebra is semiprime. A von Neumann algebra is prime if and only if it is a factor (i.e., its center consists of scalar multiples of the identity).

\section{AdDitive CENTRALIZING MAPPINGS ON VON NeUMANN ALGEBRAS}

This section is devoted to the proof of the following theorem.

Theorem 2.1. Let $R$ be a von Neumann algebra. If $F: R \rightarrow R$ is an additive centralizing mapping, then there exist $c \in Z(R)$ and an additive mapping $\zeta: R \rightarrow Z(R)$ such that $F(x)=c x+\zeta(x)$ for all $x \in R$.

For the proof of Theorem 2.1 we need several lemmas. The first lemma is implicitly contained in [4]. 
Lemma 2.2. Let $R$ be any ring and $F: R \rightarrow R$ an additive commuting mapping. Then

$$
[x, F(y)] z[u, v]=[x, y] z[u, F(v)]
$$

holds for all $x, y, z, u, v \in R$.

Proof. A linearization of $[x, F(x)]=0$ gives

$$
[x, F(y)]=[F(x), y] \quad \text { for all } x, y \in R \text {. }
$$

Replacing $y$ by $y z$ in (1) we obtain

$$
[x, F(y z)]=[F(x), y z]=[F(x), y] z+y[F(x), z],
$$

and so, using (1) again, we get

$$
[x, F(y z)]=[x, F(y)] z+y[x, F(z)] \quad \text { for all } x, y, z \in R .
$$

According to (2) we have

$$
\begin{aligned}
([x, & F(y)] z+y[x, F(z)]) u+x([u, F(y)] z+y[u, F(z)]) \\
& =[x, F(y z)] u+x[u, F(y z)] \\
& =[x u, F(y z)] \\
& =[x u, F(y)] z+y[x u, F(z)] \\
& =([x, F(y)] u+x[u, F(y)]) z+y(x[u, F(z)]+[x, F(z)] u) .
\end{aligned}
$$

Thus $[x, F(y)][z, u]=[y, x][u, F(z)]$, so it follows from (1) that

$$
[x, F(y)][z, u]=[x, y][z, F(u)] \quad \text { for all } x, y, z, u \in R \text {. }
$$

Now, since for any mapping $F$ the identity

$$
\begin{aligned}
{[x, F(y)] z[u, v]-[x, y] z[u, F(v)]=} & {[x, F(y)][z u, v]-[x, y][z u, F(v)] } \\
& -([x, F(y)][z, v]-[x, y][z, F(v)]) u
\end{aligned}
$$

holds for arbitrary $x, y, z, u, v \in R$, (3) yields the assertion of the lemma.

If $R$ is a ring, by $I([R, R])$ we denote the ideal of $R$ generated by all commutators in $R$. As a consequence of Lemma 2.2 we get

Corollary 2.3. Let $R$ be a ring with unit element 1 , such that $I([R, R])=R$. If $F: R \rightarrow R$ is an additive commuting mapping, then there exist $c \in Z(R)$ and an additive mapping $\zeta: R \rightarrow Z(R)$ such that $F(x)=c x+\zeta(x)$ for all $x \in R$. Proof. By assumption, $\sum_{i=1}^{n} z_{i}\left[u_{i}, v_{i}\right] w_{i}=1$ for some $z_{i}, u_{i}, v_{i}, w_{i} \in R$. Given $x, y \in R$, we then have by Lemma 2.2

$$
\begin{aligned}
{[x, F(y)] } & =[x, F(y)] \sum_{i=1}^{n} z_{i}\left[u_{i}, v_{i}\right] w_{i} \\
& =[x, y] \sum_{i=1}^{n} z_{i}\left[u_{i}, F\left(v_{i}\right)\right] w_{i} .
\end{aligned}
$$


Thus the element $c=\sum_{i=1}^{n} z_{i}\left[u_{i}, F\left(v_{i}\right)\right] w_{i}$ satisfies

$$
[x, F(y)]=[x, y] c \quad \text { for all } x, y \in R .
$$

We want to show that $c \in Z(R)$. From (4) it follows that

$$
\begin{aligned}
z[x, y] c+[z, y] c x & =z[x, F(y)]+[z, F(y)] x \\
& =[z x, F(y)] \\
& =[z x, y] c \\
& =[z, y] x c+z[x, y] c .
\end{aligned}
$$

Thus $[z, y][c, x]=0$ for all $x, y, z \in R$. Replacing $x$ by $x r$ and using $[c, x r]=[c, x] r+x[c, r]$ we obtain $[z, y] x[c, r]=0$ for all $x, y, z \in R$. Hence

$$
[c, r]=\left(\sum_{i=1}^{n} z_{i}\left[u_{i}, v_{i}\right] w_{i}\right)[c, r]=0
$$

for all $r \in R$, which means that $c \in Z(R)$. Now (4) can be rewritten in the form $[x, F(y)-c y]=0$ for all $x, y \in R$. Thus the mapping $\zeta(y)=F(y)-c y$ maps $R$ into $Z(R)$. The proof of the corollary is complete.

Remark 2.4. Let $S$ be any ring with unit element 1 and containing the element $\frac{1}{2}$. Let $R$ be a ring of $2 \times 2$ matrices over $S$. Then $R$ satisfies the requirements of Corollary 2.3; namely, the elements

$$
x=\left[\begin{array}{cc}
1 & 0 \\
0 & -1
\end{array}\right], \quad y=\left[\begin{array}{cc}
0 & \frac{1}{2} \\
\frac{1}{2} & 0
\end{array}\right]
$$

satisfy $-[x, y]^{2}=I$, the unit element of $R$, and so $I([R, R])=R$. This observation is the concept behind the proof of the following two lemmas.

Lemma 2.5. Let $e$ and $f$ be orthogonal, equivalent projections in a von Neumann algebra $R$. Then $R$ contains the elements $x$ and $y$ such that $\|x\| \leq 1$, $\|y\| \leq 1$, and $-[x, y]^{2}=e+f$.

Proof. By assumption, $e=u^{*} u$ and $f=u u^{*}$ for some partial isometry $u \in R$. From $u=f u e$ it follows that $u^{2}=0$. Let $x=e-f$ and $y=\frac{1}{2}\left(u+u^{*}\right)$. Using $u^{2}=0, u=u e$, and $u^{*}=u^{*} f$ we obtain $x y=\frac{1}{2}\left(u^{*}-u\right)=-y x$. Hence $[x, y]^{2}=-(e+f)$. Since $x=x^{*}$ and $x^{2}=e+f$ we see that $\|x\|=1$. Similarly, $y=y^{*}, y^{2}=\frac{1}{4}(e+f)$ and, therefore, $\|y\|=\frac{1}{2}$.

Generally, von Neumann algebras do not satisfy the hypothesis of Corollary 2.3. Namely, if $R$ is commutative, or, more generally, if it contains a nonzero $I_{1}$ summand (= largest abelian central summand), then certainly $I([R, R]) \neq$ $R$. However, the following is true.

Lemma 2.6. Let $R$ be a von Neumann algebra with no central portion of type $I_{1}$. Then $I([R, R])=R$.

Proof. First assume that $R$ has no central portion of type $I$. Then $R$ contains two orthogonal equivalent projections with sum equal to the unit element of $R$. 
By Lemma 2.5 we then have $I([R, R])=R$. Therefore we may assume that $R$ is of type $I$.

Suppose temporarily that $R$ is of type $I_{n}$ where $n$ is even or infinite. Then there exists a family $\left(e_{a}\right)_{a \in A}$ of mutually orthogonal equivalent (abelian) projections in $R$, such that the cardinal number of $A$ is $n$ and $\sum_{a \in A} e_{a}=1_{n}$, the unit element in $R$. Since $n$ is even or infinite, there exist disjoint subsets $B$ and $C$ of $A$ with the same cardinal number, such that $A=B \cup C$. The projections $e=\sum_{b \in B} e_{b}$ and $f=\sum_{c \in C} e_{c}$ are orthogonal equivalent, and their sum is $1_{n}$. By Lemma 2.5 there exist elements $x_{n}$ and $y_{n}$ such that $\left\|x_{n}\right\| \leq 1$, $\left\|y_{n}\right\| \leq 1$, and $-\left[x_{n}, y_{n}\right]^{2}=1_{n}$.

Now assume that $R$ is of type $I_{n}$ where $n=2 k+1, k \geq 1$. The unit element $1_{n}$ in $R$ is equal to the sum $e_{1}+e_{2}+\cdots+e_{2 k+1}$, where $e_{i}$ are mutually orthogonal equivalent projections. Let $p=e_{1}+e_{2}+\cdots+e_{k}$ and $q=e_{k+1}+e_{k+2}+\cdots+e_{2 k}$. Then $p$ and $q$ are orthogonal equivalent projections and so by Lemma 2.5 there exist elements $z_{n}, w_{n} \in R$ such that $\left\|z_{n}\right\| \leq 1$, $\left\|w_{n}\right\| \leq 1$, and $-\left[z_{n}, w_{n}\right]^{2}=p+q=e_{1}+e_{2}+\cdots+e_{2 k}$. Similarly we see that there exist elements $u_{n}, v_{n} \in R$ such that $\left\|u_{n}\right\| \leq 1,\left\|v_{n}\right\| \leq 1$, and $-\left[u_{n}, v_{n}\right]^{2}=$ $e_{2}+e_{3}+\cdots+e_{2 k+1}$. The element $r_{n}=-\left(e_{1}+\frac{1}{2}\left(e_{2}+e_{3}+\cdots+e_{2 k}\right)+e_{2 k+1}\right)$ then satisfies $r_{n}\left[z_{n}, w_{n}\right]^{2}+r_{n}\left[u_{n}, v_{n}\right]^{2}=1_{n}$ and $\left\|r_{n}\right\|=1$.

Now, let $R$ be any von Neumann algebra of type $I$ with no central portion of type $I_{1}$, and $\left(p_{n}\right)$ the family of mutually orthogonal central projections such that $\sum p_{n}=1$ and $p_{n} R$ is of type $I_{n}$ or $p_{n}=0$. If $n$ is odd, then there exist the elements $z_{n}, w_{n}, u_{n}, v_{n}, r_{n} \in p_{n} R$ of norm not exceeding 1 , such that $r_{n}\left[z_{n}, w_{n}\right]^{2}+r_{n}\left[u_{n}, v_{n}\right]^{2}=p_{n}$. Since all the elements are of bounded norm, we may define $r=\sum_{k=1}^{\infty} r_{2 k+1}, z=\sum_{k=1}^{\infty} z_{2 k+1}$, and analogously define the elements $w, u$, and $v$. Then $r[z, w]^{2}+r[u, v]^{2}=\sum_{k=1}^{\infty} p_{2 k+1}$. If $n$ is even or infinite then there exist elements $x_{n}, y_{n} \in p_{n} R$ such that $-\left[x_{n}, y_{n}\right]^{2}=p_{n}$ and $\left\|x_{n}\right\| \leq 1,\left\|y_{n}\right\| \leq 1$. Let $x$ and $y$ be the sum of all such $x_{n}$ and $y_{n}$, respectively. Then $-[x, y]^{2}=1-\sum_{k=1}^{\infty} p_{2 k+1}$. Thus $1=r[z, w]^{2}+r[u, v]^{2}-$ $[x, y]^{2}$, which means $I([R, R])=R$. This completes the proof.

Proof of Theorem 2.1. Since von Neumann algebras are semiprime, we may, as pointed out in the introduction, assume that $F$ is commuting.

The unit element 1 of $R$ is the sum of two orthogonal central projections $p_{1}$ and $p_{2}$ such that $p_{1} R$ is of type $I_{1}$ and $p_{2} R$ contains no central portion of type $I_{1}$. We have

$$
F(x)=p_{1} F(x)+p_{2} F\left(p_{1} x\right)+p_{2} F\left(p_{2} x\right), \quad x \in R .
$$

Note that the mapping $f: p_{2} R \rightarrow p_{2} R$ defined by $f\left(p_{2} x\right)=p_{2} F\left(p_{2} x\right)$ is commuting on $R$. By Lemma 2.6 we have $I\left(\left[p_{2} R, p_{2} R\right]\right)=p_{2} R$ and so it follows from Corollary 2.3 that $p_{2} F\left(p_{2} x\right)=f\left(p_{2} x\right)=c_{1} p_{2} x+\zeta_{1}\left(p_{2} x\right)$, where $c_{1} \in Z\left(p_{2} R\right)$ and $\zeta_{1}$ is an additive mapping of $p_{2} R$ into $Z\left(p_{2} R\right) \subseteq Z(R)$. Of course, the element $c=c_{1} p_{2}$ lies in $Z(R)$. 
By (1) we see that any commuting mapping leaves the center of ring invariant. Since $p_{1} R \subseteq Z(R)$ we then have $p_{2} F\left(p_{1} x\right) \in Z(R)$.

Now, (5) can be written as $F(x)=c x+\zeta(x)$, where $\zeta(x)=p_{1} F(x)+$ $p_{2} F\left(p_{1} x\right)+\zeta_{1}\left(p_{2} x\right)$. By above all three summands lie in $Z(R)$, thus $\zeta$ maps $R$ into $Z(R)$. The proof of the theorem is complete.

\section{Centralizing $\alpha$-Derivations}

Throughout this section $\alpha$ denotes an automorphism of a ring.

Lemma 3.1. Let $R$ be a semiprime ring and $d$ be a commuting $\alpha$-derivation of R. Then

$$
[x, y] R d(w)=0 \quad \text { for all } x, y, w \in R .
$$

Proof. Linearizing $[d(x), x]=0$ we get

$$
[d(x), y]=[x, d(y)] \text { for all } x, y \in R .
$$

In particular, $[d(x), y x]=[x, d(y x)]$ for all $x, y \in R$. On the other hand, using $[d(x), x]=0$ and $(6)$, we have

$$
[d(x), y x]=[d(x), y] x=[x, d(y)] x,
$$

and

$$
\begin{aligned}
{[x, d(y x)] } & =[x, \alpha(y) d(x)+d(y) x] \\
& =[x, \alpha(y)] d(x)+[x, d(y)] x .
\end{aligned}
$$

Comparing the above expressions we obtain $[x, \alpha(y)] d(x)=0$ for all $x, y \in$ $R$. Since $\alpha$ is onto we then also have $[x, z] d(x)=0$ for all $x, z \in R$. Replacing $z$ by $y z$ in the last relation and using $[x, y z]=[x, y] z+y[x, z]$, it follows that

$$
[x, y] z d(x)=0 \quad \text { for all } x, y, z \in R \text {. }
$$

A linearization of $(7)$ gives $[x, y] z d(w)+[w, y] z d(x)=0$ for all $x, y, z \in$ $R$. Hence

$$
\begin{aligned}
([x, y] z d(w)) R([x, y] z d(w)) & =-[x, y] z d(w) R[w, y] z d(x) \\
& \subseteq[x, y] R d(x)=0
\end{aligned}
$$

by (7). By the semiprimeness of $R$ it follows that $[x, y] z d(w)=0$ for all $x, y, z, w \in R$.

Corollary 3.2. Let $R$ be a semiprime ring. If an inner derivation $d$ of $R$ is commuting then $d=0$. If (in case $R$ has a unit element) an inner automorphism $\alpha$ is commuting then $\alpha=1$.

Proof. By assumption there exists $a \in R$ such that $d(x)=[a, x], x \in R$. By Lemma 3.1 we have $[a, x] R[a, x]=0$ for all $x \in R$, which yields $[a, x]=0$ since $R$ is semiprime.

If $\alpha$ is an automorphism then the mapping $1-\alpha$ is an $\alpha$-derivation. By assumption, $(1-\alpha)(x)=x-t x t^{-1}=[x, t] t^{-1}$ for some invertible $t \in R$. 
From Lemma 3.1 it follows that $[x, t] t^{-1} R[x, t] t^{-1}=0, x \in R$, and so $[x, t] t^{-1}=0, x \in R$. That is, $\alpha=1$.

Remark 3.3. We recall a few facts about semiprime rings $R$. Suppose that $a R b=0$ for some $a, b \in R$. In this case $b R a R b R a=0, b a R b a=0$, and $a b R a b=0$. Since $R$ is semiprime it follows that $b R a=0, b a=0$, and $a b=0$. Note that the left annihilator and the right annihilator of an ideal $U$ in $R$ coincide. It will be denoted by $\operatorname{Ann}(U)$. It can be easily shown that $U \cap \operatorname{Ann}(U)=0$, and $U \oplus \operatorname{Ann}(U)$ is an essential ideal of $R$ (recall that the ideal $U$ in a ring $R$ is essential if $U \cap I \neq 0$ for every nonzero ideal $I$ in $R$ ).

Our next purpose is to discuss commuting $\alpha$-derivations on semiprime rings. First we show a simple example of a nonzero commuting derivation on a noncommutative semiprime ring. Take $C$ to be a commutative semiprime ring with a nonzero derivation $\delta$. If $S$ is any noncommutative semiprime ring, then $R=C \oplus S$ is again a noncommutative semiprime ring. The mapping $d: R \rightarrow R$, $d(c, s)=(\delta(c), 0)$ is then a nonzero commuting derivation. Roughly speaking, we will show that this example is in fact the only possible example.

H. E. Bell and W. S. Martindale [2] studied centralizing endomorphisms and centralizing derivations on semiprime rings. In fact they merely assumed that the mappings are centralizing on some nonzero one-sided ideal of a semiprime ring. As a result they obtained the existence of a nonzero central ideal. In our next theorem we obtain (of course, under stronger assumptions) the existence of two ideals: one is central and on the other one the commuting mapping vanishes.

Theorem 3.4. Let $R$ be a semiprime ring and let $d: R \rightarrow R$ be a commuting $\alpha$-derivation. Then there exist ideals $U$ and $V$ of $R$ such that:

(i) $U \cap V=0$ and $U \oplus V$ is an essential ideal of $R$,

(ii) $\alpha$ maps $U$ onto $U$ and $V$ onto $V$,

(iii) $V \subseteq Z(R)$ and $d$ maps $R$ into $V$,

(iv) $d=0$ on $U$.

Proof. Let $V=\operatorname{Ann}(I([R, R]))$ and $U=\operatorname{Ann}(V)$. Thus (i) holds by Remark 3.3.

Obviously $\alpha$ maps $I([R, R])$ onto itself. Given $v \in V, u \in I([R, R])$ we have $0=\alpha(v u)=\alpha(v) \alpha(u)$. Since $\alpha(u)$ is an arbitrary element in $I([R, R])$ it follows that $\alpha(v) \in \operatorname{Ann}(I[R, R])=V$; that is, $\alpha$ maps $V$ into itself. Analogously $\alpha^{-1}$ maps $V$ into itself. This means that $\alpha$ is onto on $V$. In a similar fashion one proves that $\alpha$ maps $U$ onto itself. Thus (ii) is proved.

By the definition of $V, v R[x, y]=0$ for all $v \in V, x, y \in R$. Hence $[v, y] R[v, y]=0$ for all $v \in V, y \in R$. Since $R$ is semiprime it follows that $[v, y]=0$. Thus $V \subseteq Z(R)$. By Lemma 3.1 (and Remark 3.3) we see that $d(w) \in V$ for any $w \in R$. This proves (iii).

Given $u \in U, v \in V$ we have $0=d(v u)=\alpha(v) d(u)+d(v) u$. By (iii), $d(v) \in V$ and so $d(v) u=0$. Hence $\alpha(v) d(u)=0$ for all $v \in V, u \in U$. 
Since $\alpha$ maps $V$ onto $V$ it follows that $d(u) \in \operatorname{Ann}(V)=U$. But we have proved that $d$ maps $R$ into $V$; thus $d(u) \in U \cap V=0$. The proof is complete.

C. R. Miers proved that if $\alpha$ is a centralizing *-automorphism of a von Neumann algebra $R$, then $R=R_{1} \oplus R_{2}$ where $R_{1}$ is commutative and $\alpha$ is the identity on $R_{2}$ [18, Theorem 5]. He pointed out that it would be desirable to prove this result without the assumption that $\alpha$ preserves adjoints (verifying this for the special case that $\alpha$ is inner). We do this now.

Corollary 3.5. If $\alpha$ is a centralizing automorphism of a von Neumann algebra $R$, then there exists a central projection $p$ in $R$ such that $\alpha(p)=p$, the ideal $p R$ is commutative, and $\alpha$ is the identity on $(1-p) R$.

Proof. Since $R$ is semiprime, $\alpha$ is commuting. Set $d=1-\alpha$ and apply Theorem 3.4. Since the ideal $V$ is the annihilator of an ideal, it follows that $V$ is weak-operator-closed. Hence $V=p R$ for some central projection $p$. But then $U=\operatorname{Ann}(V)=(1-p) R$. The statement (ii) in Theorem 3.4 means that $\alpha(p)=p$. The proof is complete.

We note that Corollary 3.5 holds with an analogous proof in a more general case where $R$ is a semiprime Baer ring (see [11, Theorem 13]).

Remark 3.6. Let us present another proof of Corollary 3.5. There exists a central projection $p$ in $R$ such that the ideal $p R$ is commutative and the ideal $(1-p) R$ contains no central portion of type $I_{1}$. By Lemma 3.1 we have $I([R, R])(w-\alpha(w))=0$ for all $x, y, w \in R$. According to Lemma 2.6 $1-p \in I([R, R])$ and so $(1-p)(w-\alpha(w))=0$ for all $x, y, w \in R$. Since an automorphism takes commutative ideals of $R$ onto commutative ideals it follows that $\alpha(p R)=p R$; that is, $\alpha(p)=p$. Thus $(1-p)(w-\alpha(w))=0$ means that $\alpha((1-p) w)=(1-p) w$. This proves the corollary.

In [5] it was shown that any continuous commuting (Jordan) derivation of a Banach algebra $A$ maps the algebra into its radical $\operatorname{rad}(A)$. Theorem 3.4 enables to obtain the analogous result for derivations on semiprime Banach algebras without assuming the continuity. In [5] we used the different approach; namely, the proof based on a theorem of A. M. Sinclair [20], which states that every continuous derivation of a Banach algebra $A$ leaves the primitive ideals of $A$ invariant.

Corollary 3.7. Let $d$ be a centralizing derivation of a semiprime Banach algebra $A$. The $d$ maps $A$ into $Z(A) \cap \operatorname{rad}(A)$.

Proof. Again we may assume that $d$ is commuting. By Theorem $3.4 d$ maps $A$ into the ideal $V \subseteq Z(A)$. Consider the restriction of $d$ to $V$. Since $V$ is the annihilator of some set we see that $V$ is closed; thus $V$ is a commutative Banach algebra. By a theorem of $\mathrm{M}$. Thomas [21] $d$ maps $V$ into $\operatorname{rad}(V)$. Given $x, y \in A$, we have $d(x) \in V, d(x) y \in V$ and, therefore, $d(d(x))=d^{2}(x) \in$ $\operatorname{rad}(V), d(d(x) y)=d^{2}(x) y+d(x) d(y) \in \operatorname{rad}(V)$. Consequently $d(x) d(y) \in$ $\operatorname{rad}(V)$ for all $x, y \in A$. But then we also have $d(x) y d(x)=d(x) d(y x)-$ $d(x) d(y) x \in \operatorname{rad}(V) \subseteq \operatorname{rad}(A)$. The algebra $A / \operatorname{rad}(A)$ is semisimple and, 
therefore, also semiprime. Hence the relation $d(x) y d(x) \in \operatorname{rad}(A)$ implies that $d(x) \in \operatorname{rad}(A)$. The proof of the corollary is thus complete.

\section{Centralizing antihomomorphisms}

Note that the involution $*$ on a ring of quaternions is a commuting antihomomorphism. Recall that $x \in Z(H)$ if and only if $x=x^{*}$. With this example in mind we state the following result.

Proposition 4.1. Let $R$ be a 2-torsionfree semiprime ring and let $\theta: R \rightarrow R$ be a centralizing antihomomorphism. Then $S=\{x \in R \mid x=\theta(x)\} \subseteq Z(R)$. Moreover, if $R$ is prime and $\theta$ does not map $R$ into $Z(R)$ then $S=Z(R)$. Proof. Since $R$ is semiprime and 2-torsionfree, $\theta$ is commuting. A linearization of $[x, \theta(x)]=0$ gives

$$
[x, \theta(y)]+[y, \theta(x)]=0 \quad \text { for all } x, y \in R .
$$

Put $y z$ instead of $y$ in (8). Then we obtain

$$
\begin{aligned}
0 & =[x, \theta(z) \theta(y)]+[y z, \theta(x)] \\
& =\theta(z)[x, \theta(y)]+[x, \theta(z)] \theta(y)+y[z, \theta(x)]+[y, \theta(x)] z
\end{aligned}
$$

and according to $(8)$ we arrive at

(9) $y[z, \theta(x)]+[y, \theta(x)] z=\theta(z)[y, \theta(x)]+[z, \theta(x)] \theta(y)$

for all $x, y, z \in R$.

Take $x \in S$ and write $x$ for $z$ in (9). Then we get $[y, x] x=x[y, x]$ for all $y \in R$ and hence $x \in Z(R)$ by [8, Lemma 1.1.9]. Thus $S \subseteq Z(R)$.

Now let $R$ be prime and take $z \in Z(R)$. By (9) we have $(\theta(z)-z)[y, \theta(x)]$ $=0$ for all $x, y \in R$. Replacing $y$ by $y z$ and using $[y z, \theta(x)]=[y, \theta(x)] z+$ $y[z, \theta(x)]$ we get $(\theta(z)-z) y[w, \theta(x)]=0$ for all $x, y, w \in R$. By assumption there exists $x \in R$ such that $\theta(x) \notin Z(R)$ and so the last relation implies $\theta(z)=z$ since $R$ is prime. Consequently $S=Z(R)$. The proof is complete.

\section{ACKNOWLEDGEMENT}

I am indebted to Dr. B. Magajna for his valuable suggestions.

\section{REFERENCES}

1. R. Awtar, Lie and Jordan structures in prime rings with derivations, Proc. Amer. Math. Soc. 41 (1973), 67-74.

2. H. E. Bell and W. S. Martindale, Centralizing mappings of semiprime rings, Canad. Math. Bull. 30 (1987), 92-101.

3. $\ldots$, Semiderivations and commutativity in prime rings, Canad. Math. Bull. 31 (1988), 500-508.

4. M. Brešar, Centralizing mappings and derivations in prime rings, preprint.

5. M. Brešar and J. Vukman, On left derivations and related mappings, Proc. Amer. Math. Soc. 110 (1990), 7-16. 
6. 178-185.

7. L. O. Chung and J. Luh, On semicommuting automorphisms of rings, Canad. Math. Bull. 21 (1978), 13-16.

8. I. N. Herstein, Rings with involution, University of Chicago Press, Chicago, 1976.

9. Y. Hirano, A. Kaya and H. Tominaga, On a theorem of Mayne, Math. J. Okayama Univ. 25 (1983), 125-132.

10. R. V. Kadison and J. R. Ringrose, Fundamentals of the theory of operator algebras, vol. 1, Academic Press, London, 1983; vol. 2. Academic Press, London, 1986.

11. I. Kaplansky, Rings of operators, Benjamin, New York, 1968.

12. C. Lanski, Differential identities, Lie ideals, and Posner's theorems, Pacific J. Math. 134 (1988), 275-297.

13. W. S. Martindale, Prime rings satisfying a generalized polynomial identity, J. Algebra 12 (1969), 576-584.

14. M. Mathieu, Elementary operators on prime $C^{*}$-algebras I, Math. Ann. 284 (1989), 223244.

15. J. Mayne, Centralizing automorphisms of prime rings, Canad. Math. Bull. 19 (1976), 113115.

16. _ Ideals and centralizing mappings in prime rings, Proc. Amer. Math. Soc. 86 (1982), 211-212; Erratum 89 (1983), 187.

17. _ Centralizing mappings of prime rings, Canad. Math. Bull. 27 (1984), 122-126.

18. C. R. Miers, Centralizing mappings of operator algebras, J. Algebra 59 (1979), 56-64.

19. E. C. Posner, Derivations in prime rings, Proc. Amer. Math. Soc. 8 (1957), 1093-1100.

20. A. M. Sinclair, Continuous derivations on Banach algebras, Proc. Amer. Math. Soc. 20 (1969), 166-170.

21. M. Thomas, The image of a derivation is contained in the radical, Ann. Math. 128 (1988), 435-460.

Institute of Mathematics, Physics and Mechanics, University of Ljubljana, P. O. Box 543, 61111 LuUbluana, Yugoslavia 